\title{
Überlegungen zu den Funktionen der Okkasionalismen im deutschen und ägyptisch-arabischen Sprachgebrauch
}

\author{
Rasha Emadeldin Sayed Mohamed
}

Abteilung für Germanistik der Al-Alsun Fakultät der Minia Universität rascha_nar@yahoo.com

Received: May 22, 2020 Accepted: August 30, 2020 Published: October 26, 2020

\begin{abstract}
In allen lebendigen Sprachen sterben manche Wörter aus und werden immer wieder neue Wörter erfunden oder von alten Wörtern neu gebildet. Diese Wortschatzerweiterung gilt als natürlichen Prozess der Sprachen, um mit den neuen Veränderungen der Welt und des Lebens Schritt zu halten sowie um neue Gegenstände, Produkte, Erscheinungen, Entwicklungen ... usw. zu beschreiben und zu benennen.
\end{abstract}

Der vorliegende Beitrag befasst sich mit den okkasionellen Bildungen im deutschen und ägyptisch-arabischen Sprachgebrauch und setzt sich mit den pragmatischen Aspekten der Okkasionalismen, ihrem Gebrauch und ihren Auswirkungen in der Kommunikation auseinander. Vor diesem Hintergrund behandelt dieser Beitrag die folgenden zentralen Fragestellungen:

1. Wie und warum verwendet man diese Wortschöpfungen statt den usuellen lexikalisierten Bildungen?

2. Welche Funktionen üben die Okkasionalismen im deutschen und ägyptisch-arabischen Sprachgebrauch aus? Und welche Effekte kann der Sprecher erzielen, wenn er diese Bildungen gebraucht?

Damit setzte ich mir zum Ziel, den Begriff Okkasionalismus im Deutschen näher zu erläutern und die Funktionen und Verwendungen der Okkasionalismen in ihren konkreten Kontexten im Deutschen und im Ägyptisch-Arabischen zu verstehen und zu analysieren.

Schlüsselwörter: Okkasionalismen, Ad-hoc-Bildungen, Augenblicks-, 
Gelegenheits-, Wortneubildungen, Wortschöpfungen

\section{Einleitung}

In allen lebendigen Sprachen befinden sich zahlreiche neu gebildete Wörter, die nur aus einer speziellen Situation herausentstanden sind und noch nicht in den allgemein bekannten Wortschatz einer Sprache aufgenommen werden. Die Wörter wie Nureinbiertrinker, Spuckling, blumengleich, gegengoogeln, strafbelobigt, und يهبيد yahbid (dt. quatschen), ألش 'alš (dt. Spott), نبيسى nepepsi (dt. lass uns Pepsi


الكأكititkawkaw fí 'alkākāw (dt. mit Kakaohergestellte Kekse) ...usw. sind spontane und oft einmalige Wortneubildungen und hinsichtlich ihrer Struktur auffällig und einmalig, da sie sich auf eine bestimmte Situation beziehen und spontan vom Sprecher gebildet werden, um bestimmte Funktionen zu erfüllen. Es handelt sich dabei um die so genannten Okkasionalismen (bzw. Ad-hoc-Bildungen, Augenblicks-, Gelegenheitsbildungen).

Den Gegenstand des vorliegenden Beitrags stellen die Okkasionalismen in der deutschen und ägyptischen Gegenwartssprache dar. Im Rahmen dieses Beitrags soll zwischen Okkasionalismen, Neologismen und usuellen Lexemen differenziert werden. Der Beitrag stellt sich die Aufgabe, das Phänomen „Okkasionalismus“ “ im Deutschen nach seinem begrifflichen Inhalt zu erklären und zu verstehen und die okkasionellen Wörter in beiden Sprachen vorzustellen, um ihre Funktionen in ihren konkreten Kontexten zu untersuchen.

Der vorliegende Beitrag gliedert sich in drei Hauptteile. Der erste und zweite Teil widmen sich jeweils der terminologischen Klärung des Begriffs „Okkasionalismus“ “ im Deutschen.

Der Gegenstand und Ziele des Beitrags werden auch aufgezeigt. Es wird die Definition der Okkasionalismen im Deutschen und eine Folge von okkasionellen Wörtern in verschiedenen Formen und Texten dargestellt. Nachfolgend beziehe ich mich auf das Ägyptisch-Arabische als gesprochene Umgangssprache in dem dritten Teil, da das Phänomen von Okkasionalismen keine bestimmte Wiedergabe im Hocharabischen hat, 
sondern es vor allem der gesprochenen Sprache angehört. Die ägyptische Umgangssprache zeichnet sich durch ihren Reichtum des Wortschatzes und durch ihre zeitliche und räumliche Ausdehnung aus. Sie umfasst nicht nur das traditionelle und klassische Hocharabische in ihren verschiedenen Entwicklungsstadien sondern wird auch von anderen Sprachen beeinflusst. Die ägyptische Sprache neigt zum Sarkasmus und zu der unerschöpflichen Kreativität. Die Okkasionlismen im ÄgyptischArabischen tauchen heutzutage immer mehr in der Werbung, da sie dank ihrer ungewöhnlichen Struktur bestimmte Effekte beim Rezipienten erzielen. Gelegentlich beziehe ich mich auf einige ägyptische konkrete Beispiele aus dem Alltag zur Darstellung des analytischen Phänomens. Daher stellt der dritte letzte Teil des Beitrags die Funktionen der Okkasionalismen im deutschen und im ägyptisch-arabischen Sprachgebrauch an und fasst die Schlussfolgerungen des Artikels zusammen.

\section{Zum Begriff „Okkasionalismus““}

Der Begriff Okkasionalismus lässt sich vom lateinischen Wort occasio ableiten, das Gelegenheit oder Anlass bedeutet (vgl. Ulrich, 2002, S. 205). Die Okkasionalismen (bzw. Ad-hoc-Bildungen, Einmal-, Augenblicks-, Gelegenheitsbildungen, Textwörter) entstehen nur für einen bestimmten Zweck aus einer speziellen Situation oder für einen konkreten Text bzw. einen bestimmten Kontext heraus (vgl. Glück \& Rödel, 2016, S. 227). Ohne diesen Kontext zu wissen sind sie schwer interpretierbar. Solche Bildungen werden in der Regel nicht ins Lexikon aufgenommen, sondern verschwinden wieder, sobald die Situation vorbei ist.

Durch diese Abbildung verdeutlicht Fellner (2009, S. 5), wie die Okkasionalismen durch ihre Spontanität, Häufigkeit, Kontextabhängigkeit und ihre Kurzlebigkeit ausgezeichnet sind. Die sprachlichen Gelegenheitsbildungen heben die Merkmale der Kurzlebigkeit und Spontanität von Okkasionalismen hervor und die Bezeichnung der Einmaligkeit von Okkasionalismen betont ihren einmaligen und ganz seltenen Gebrauch. Ausgehend davon, dass sie von einem bestimmten Kontext abhängen und auf die textuellen Funktionen beruhen, werden sie als Textwörter bezeichnet. 
Die Okkasionalismen haben die Eigenschaft, einen neuen komplexen Sachverhalt in einem einzigen Wort im Augenblick des Sprechens auszudrücken. Demzufolge kommen sie immer wieder gern in Diskussionen, Zeitungen, in der Propaganda oder Werbesprache auf, wobei der Kontext eine große Rolle spielt. Falls die okkasionelle Bildung mehrmals gebraucht wird und dem Wortschatz der Sprache angehört sowie im Sprachgebrauch und Lexikon als neues Wort etabliert wird, ist sie als Neologismus zu werten und nicht mehr als Okkasionalismus (Harm, 2015, S. 119). Außerdem werden die okkasionellen Wörter sich durch die wiederholte regelmäßige Verwendung zu der usuellen Wortbildung weiterentwickeln. Der Hauptunterschied dazwischen bestätigt Bußmann darin,

In der Wortbildung [unterscheidet man] zwischen (nach produktiven Wortbildungsregeln geschaffenen) Neubildungen, die spontan aus einem momentanen Bedarf heraus und in starker Kontextabhängigkeit entstehen, und im Lexikon kodifizierten, zum lexikalischen Inventar einer Sprache gehörenden Ausdrücken. (Bußmann, 2008, S. 492)

So könnte z. B. kleiner Spuckling ein Säugling bezeichnen, ,der immer viel zu hastig trinkt und einen Teil der Milch wieder ausspuckt ${ }^{\text {‘i }}$. Das Beispiel wird einmalig im Moment der Kommunikation geschaffen, um den Säugling in dieser Situation zu bezeichnen. Würde dieses Wort von den Sprachbenutzern als neu empfunden und seinen Weg gerade in Wörterbüchern finden, könnte es sich zu einem Neologismus entwickeln. Wenn es zusätzlich später mehrmals benutzt, ohne diesen Kontext leicht entschlüsselt und in den Wortschatz der Sprachteilnehmer aufgenommen wird, wird es als usuelles Wort bezeichnet oder einfach wieder vergessen. In diesem Rahmen soll hinterfragt werden, warum der Sprecher die okkasionellen Bildungen anstatt der usuellen auswählt. Eine vollständige logische Antwort auf diese Frage kann folgendermaßen lauten: Die Okkasionalismen werden durch verschiedene Auswirkungen und Funktionen charakterisiert. Dazu fasst Elsen die Charakteristik und die Funktionen der Okkasionalismen darin zusammen:

Okkasionalismen (Einmal-, Augenblicks-, Gelegenheits-, Ad-hoc-Bildungen) sind häufig nur im Kontext verständlich und übernehmen oft textrelevante Aufgaben. Sie üben sprachökonomische oder verschiedene stilistische Funktionen aus und füllen lexikalische Lücken. Sie können sich zu den eigentlichen Neologismen und schließlich zu etablierten Wortschatzeinheiten 
entwickeln oder vorher wieder verschwinden. (Elsen, 2011, S. 21)

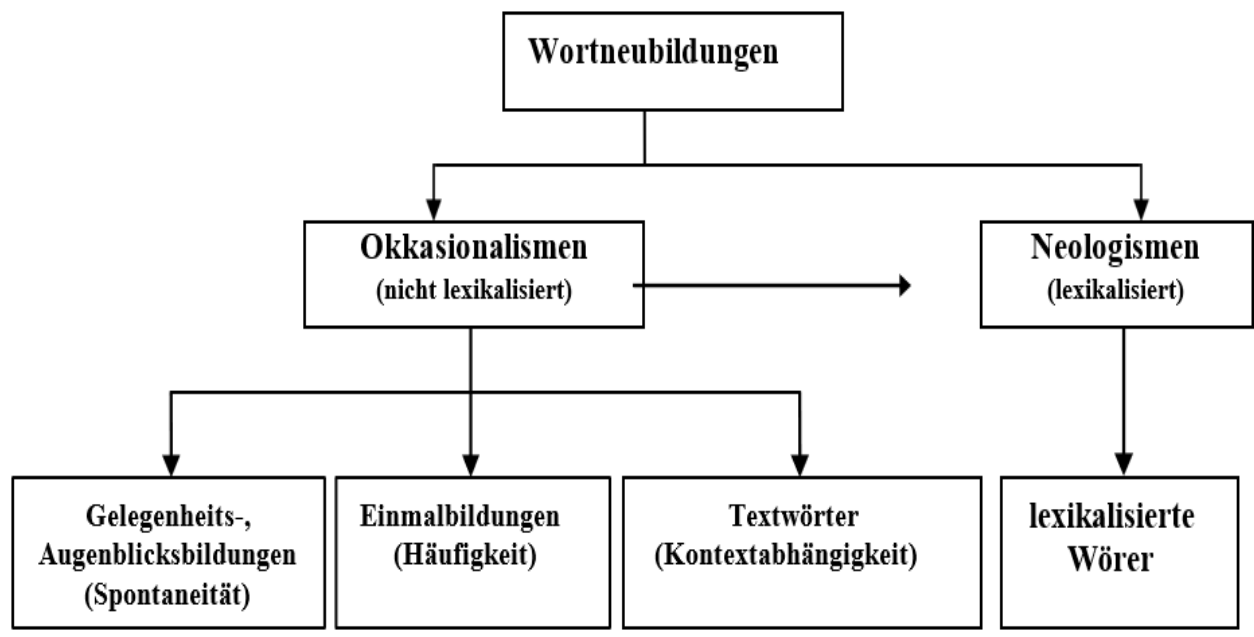

Abbildung 1: Klassifikation der Okkasionalismen (Fellner, 2009, S. 5)

Es ist sinnvoll, einen Blick auf die allgemeinen Funktionen der okkasionellen Wortneubildungen an sich zu werfen.

\section{Allgemeine Funktionen von Okkasionalismen}

Ortner fasst die pragmatischen Aspekte für die

Kompositionsneubildungen in drei kommunikativen Motiven zusammen:

Die okkasionellen Wortneubildungen werden zum einen aus „sachbezogenen Gründen“" gebildet, um unbekannte Sachverhalte in bestimmten Textsorten zu benennen. Dazu gehören z. B. ,,in lyrischen Texten als Beschreibung "innerer Welten", die immer wieder neu - auch mit lexikalischem Material - versprachlicht werden müssen, um nicht der Gefahr der Konventionalisierung zu verfallen“"(Ortner, 1984, S. 168f). Zum Weitern liegen die ,sprachsystematischen Motive“، bzw. „Analogieprotzesse“، vor, um einen neuen Aspekt am bestimmten bekannten Wort herauszustellen (Blumengeschäft/ Blumenladen: Blumenstudio). Solche pragmatischen Motive führen zum anderen zur Tendenz, ,leicht handhabbare Textelemente zu schaffen statt schwerfällige Wortgruppen zu verwenden“, ebenso zur „,Lust am Verfremdungseffekt" " und letztlich zum ,,bewussten Einsetzen von spielerischen, ironischen oder expressiven Sprachmitteln“ “ (Ortner, 1984, S. 170). 
Magdalena Matussek (1994) bestimmt in ihrem Beitrag

„Wortneubildungen im Text" für die Wortneubildungen ebenso drei Funktionen: Benennungsfunktionen, Sprachökonomie und Textfunktion.

Elsen (2011, S. 87) bezeichnet die Benennungsfunktion als referentielle Funktion. Dabei werden die Wörter, die diese Funktion ausüben sowie Gegenstände und Sachverhalte benennen und spezifizieren, ,weitgehend ohne Textzusammenhang verständlich". Wenn es einen textuellen Zusammenhang gibt, handelt es sich um eine textuelle Funktion. Elsen beschreibt die textuelle Funktion von Wortneubildungen aus verschiedenen Blickwinkeln wie folgt:

Formal gesehen verweisen Wörter auf andere Wörter. Sie ersetzen andere Wörter um Wiederholungen oder komplexe Phrasen zu vermeiden und damit die Sätze zu verkürzen, z.B. Truppenrückzug statt Rückzug der Truppen. Inhaltlich gesehen bauen sie den Wissenstand aus und/oder bündeln ihn. Sie dienen der Informationsverdichtung, der Verbildlichung, dem Kontrast. Sie ergänzen oder differenzieren Informationen oder erleichtern das Verständnis [...]. Thematisch gesehen konstituieren sie ein Thema, halten es, variieren es, kombinieren mehrere Themen oder tragen die Leitmotivik [...]. (Elsen, 2011, S. 87f.)

Elsen vertritt die Ansicht, dass die stilistische Funktion die dritte wichtigste Funktion ist, die die Neubildungen ausüben, besonders weil sie ,bestimmte Effekte beim Leser“ auslösen (Elsen, 2011, S. 88). Im Folgenden möchte ich die Funktionen der Okkasionalismen näher eingehen, die in der umfangreichen Definition von Bußmann unter dem Stichwort „Ad-hoc-Bildung” vorkommen:

Spontane, meist kontextgebundene Wortneubildung zur Bezeichnung eines neuen oder bisher nicht benannten Sachverhalts bzw. zum Ausdruck der spezifischen Einschätzung eines Referenten durch den Sprecher. A. entstehen durch kreative Anwendung von Wortbildungsregeln auf Einheiten des Lexikons, sie haben unterschiedliche textspezifische Funktionen, wie z.B.

Informationskonzentrierung [...], Ausgleich von Bezeichnungslücken [...] oder stilistische Effekte, wie sie bei bestimmten Autoren oder Medien zu finden sind. In der Regel entscheidet die statistische Häufigkeit der Wiederverwendung solcher Bildungen über ihren gleitenden Übergang zum Neologismus bzw. kodifizierten Eintrag im Lexikon. (Bußmann, 2008, S. 6)

Ich lehne mich an bestimmte Beispiele mit elaborierten okkasionellen Wortbildungen in konkreten Situationen in der deutschen und ägyptischen Umgangssprache an, um ihre Funktionen deutlich zu erklären.

\subsection{Benennungsfunktion}

In erster Linie werden die Okkasionalismen gebildet, um lexikalische 
Leerstellen zu füllen und neue Sachverhalte zu benennen bzw. zu bezeichnen, die keinen eigenen Namen haben. Demzufolge befriedigen die okkasionellen Bildungen einen kommunikativen ,permanente[n]“ Bedarf nach Ausfüllung einer Benennungslücke oder nach Variation einer vorhandenen Benennung (vgl. Duden, 2009, S. 639). Es ist eine kommunikative Notwendigkeit und ein dringender Bedarf, neue, treffende und einprägsame Bezeichnungen und Benennungen für die neuen Erscheinungen und Sachverhalte zu finden. Das lässt sich damit begründen, dass diese Bildungen sich an kulturellen, sozialen, ökonomischen, politischen und technischen Veränderungen und an dem Fortschritt in den einzelnen Fachwissenschaften anpassen (vgl. Duden, 2009, S. 641).

Das beste Beispiel für dieses Phänomen liefert der Okkasionalismus Schaulästige. ,Schaulästige“ "bringt die unangenehmen, störenden und lästigen Gaffer zum Ausdruck, die beispielsweise bei Unglücken, Unfällen, Katastrophen beobachten, den Verkehr und die Rettungsmaßnahmen behindern und manchmal auch Handyfotos von Opfern machen. Es wurde dadurch gebildet, indem zwei Wörter Schaulustige und lästig miteinander kombiniert werden, um ein neues Wort zu formen, das in der Regel kürzer und damit auch praktischer ist. In diesem Zusammenhang darf man auch nicht unerwähnt lassen, dass dieses Wort „Schaulästige“ bei der Wahl zum deutschen Wort des Jahres 2019 von Gesellschaft für deutsche Sprache (GfdS) auf dem vierten Platz $\mathrm{kam}^{\mathrm{ii}}$.

Anhand dieses Beispiels wird deutlich, dass die Okkasionalismen dazu dienen, in einer bestimmten Kommunikationssituation komplexe Sachverhalte möglichst kurz auszudrücken, da die funktionale Hauptcharakteristik der Okkasionalismen in der Sprachökonomie liegt. Beispielhaft ist hier der Okkasionalismus ,Respektrente“ des Jahres 2019 von (GfdS) gekürt wurde. Man kann nicht leugnen, dass diese okkasionelle Bildung eine lange Erklärung erspart; und zwar: ,die geplante Einführung einer Grundrente für Männer und Frauen, die trotz langjähriger Erwerbstätigkeit bisher nur eine sehr geringe Rente beziehen. Das gilt als Respekt vor den Renten ${ }^{\text {‘iii }}$. 
Es gibt auch in der ägyptischen Umgangssprache einige Jugendwörter, die sich in unserer heutigen Zeit auf große und gefährliche Weise verbreitet haben. Diese Wörter gehören nicht zum Arabischen, einige sind von der englischen Sprache entlehnt und einige haben keinen Ursprung. Auch wenn diese Wörter aus dem Arabischen entstanden, üben sie verschiedene sprachliche Verwendungen in bestimmten Kontexten. افتكاسة Das zeigt das Beispiel افتكاسة 'ftikāsah bedeutet die neue und kreative Handlung bzw. die mentale Erfindung. Es wird als eine bessere Handlungsweise in schwierigen Situationen gesagt. Das Wort hat aber eine lange Geschichte; die Wortherkunft kann فقس faqas (dt. brüten) sein. Wenn jemand bei einer hässlichen Handlung erwischt wird, sagt man zu ihm: فقستك faqastak (dt. offenlegen). In diesem Zusammenhang gibt es ein Sprichwort, das lautet, dass das Küken seine Mutter, die Henne, nicht täuschen kann, weil sie es ausbrütete. Daher wird die Person, die auf naive Weise lügt, مفقوس mafqūs genannt. Auf diese Weise bedeutet das Wort فقس faqas die Entdeckung oder das Austritt aus der Dunkelheit ins Licht. Da der Buchstabe $q \bar{a} f$ schwer beim Artikulieren ist, verwandelt es sich in $k \bar{a} f$. Das neugebildete Wort فكس fakas ist aus dieser Situation herausentstanden und das Verb افتكس aftakas bedeutet erfinden sowie muftakas äußert, was erfunden wurde und unnachahmlich ist.

جديد “ Daraus wurde ein bestimmtes Produkt in einer Fernsehwerbung als ي "neu, erfinderisch, köstlich wie kein zweites“ “ beschrieben ${ }^{\mathrm{iv}}$.

Andere Belege besagen aber, dass der Okkasionalismus فاكِس fäkis durch Verzerrung des englischen Wortes fake (Fälschung) entstanden wurde, was Fälschung oder Unwirklichkeit bedeutet. Dieses Wort hat sich unter jungen Menschen stark verbreitet und wird in bestimmten Situationen verwendet, wenn man etwas Falsches vor sich sieht oder wenn man nichts tun möchte, sagt man فاكس fäkis.

Der ägyptische Okkasionalimus ألش خانة 'alš ḩānah ist ein satirisches politisches Programm. Der Name des Programms kommt aus dem Okkasionlismus ألش 'aľ̌, was Sarkasmus bedeutet, und aus dem türkischen Wort خانة hānah, einem Ort, daher ist die Bedeutung "ein Ort 
des Sarkasmus".

\subsection{Textuelle Funktion}

Wie schon erwähnt, werden die Okkasionalismen für einen bestimmten Text gebildet und verbinden sich daran weitgehend, um textrelevante Funktionen zu übernehmen (vgl. Barz, 2007, S. 181 / Elsen, 2011, S. 21).

Das lässt sich gut am folgenden Beispiel veranschaulichen. Der Artikel über Traumkörper wurde mit dem Titel „Hammerkörper in acht Wochen” versehen (vgl. Zeitmagazin, 2017).

\section{Schlagzeile: „Hammerkörper in acht Wochen“ (14. JULI 2017)}

Untertitel: Unser Autor hatte einen Traum: Endlich sein T-Shirt ausziehen können, ohne dass einer lacht. Deshalb hat er sein Leben radikal geändert. Wurde sein Traum wahr?

Äquivalente im Text: - den raschen Weg zum Traumkörper gefunden zu haben - fit werden in ein paar Wochen - wie mein Körper aussieht, was er wiegt, wie schnell ich außer Atem gerate? Was wäre, wenn ich für einen überschaubaren Zeitraum mein Leben komplett umstellte? Wenn ich sagen wir, zwei Monate lang - alles auf die Spitze triebe, sämtliche Gesundheitstipps befolgte, mein gesamtes Leben dem Fitnesswahn unterordnete - Ich will versuchen, der fitteste Mensch auf Erden zu werden. Zwei Monate lang.

Der Okkasionalismus „Hammerkörper" ist nicht leicht außer seinem Kontext richtig zu deuten, daher wird vom Leser erwartet, seine Aufmerksamkeit auf den ganzen Text zu lenken. Hier handelt es sich um eine Gelegenheitsbildung, die in diesem Artikel über den fitten Körper entsteht. Auf den ersten Blick auf die Überschrift begegnet dem Leser eine sehr vage wirkende Gelegenheitsbildung. Durch das Textlesen findet man folgende Wörter, die dem Leser beim Verstehen des Okkasionalismus helfen; Traumkörper, der fitteste Mensch auf Erden. Nach dem Lesen des ganzen Textes gelingt es dem Leser, das Wort zu verstehen; und zwar der Hammerkörper beschreibt den fitten, schönen und attraktiven Traumkörper.

Einem wichtigen Punkt soll große Aufmerksamkeit gewidmet werden, dass der Text ungefähr aus 250 Wort besteht, in dem das Wort „Hammer" niemals außer in der Schlagzeile erwähnt wird. Auf diese 
Weise werden die Gedanken des Lesers eingeführt, um ihn dazu zu bringen, die Nachricht zu öffnen und weiterzulesen.

Wie gesehen hat das Verwenden der Okkasionalismen in Texten die Möglichkeit, den Lesevorgang anzutreiben, ein gewisses

Spannungsgefühl beim Leser zu evozieren und die Aufmerksamkeit des Lesers in Bezug auf den Textverlauf zu steuern (vgl. Fellner, 2009, S. 29). Diese textuelle Rolle der Okkasionalismen besteht darin, andere Wörter zu ersetzen, um Wiederholungen oder komplexe Phrasen zu vermeiden, um dadurch die Sätze möglichst zu kürzen. Sie dienen der Informationsverdichtung und fangen das Interesse des Lesers, insbesondere weil sie die Bezeichnungen sensationell erscheinen lassen.

Der deutsche Titel eines Artikels namens „1600 Euro fürs Faulenzen? warum eine Kunsthochschule Stipendien fürs Nichtstun vergibt “ (Tagesticket - Der Früh-Podcast · 2020-08-28)v wurde im Arabischen so

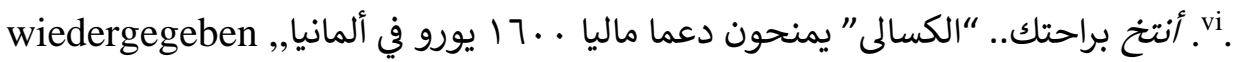
Das okkasionelle Wort أنتخ في أن 'antah ist ein moderner Ausdruck, der in den letzten Jahren verwendet wurde, insbesondere unter Jugendlichen. Was mit diesem Wort gemeint ist, ist faulenzen, faul sein, nichts tun und Flucht vor der Arbeit.

Ein weiteres Beispiel dafür liefert folgender Text ${ }^{\mathrm{vii}}$ :

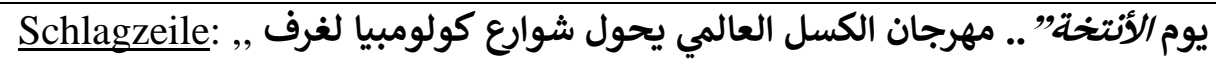
(8) 23. August 2018)

Ü.: Tag der Faulheit.. das Internationale Faulheit-festival verwandelt die Straßen Kolumbiens in Schlafzimmer

Äquivalente im Text: “اليوم العالمي للكسل "Welttag der Faulheit - مقابل اليومل Es ist gegen den Internationalen Tag der Arbeit غير الاحتفالات يوجد غناء وموسيقى ورقص وطعام من أجل الاسترخاء وتعلم الاستمتاع مقائ außer Feierlichkeiten gibt es ebenfalls Gesang, Musik, Tanz und Essen zum Entspannen, zur Vergnügung und zur Unterhaltung

Genauso wie im Deutschen kommt der Okkasionalismus in der Überschrift des Textes nur als einziges Mal vor, um die Aufmerksamkeit des Lesers auf eine bestimmte Botschaft anzuziehen, dann hilft der ganze Text beim Verstehen des Wortes. „Es handelt sich dabei um Vorgriffe 
auf Informationen, die im Fließtext explizit werden“ “ (Krieg, 2005, S. 86). Für die ältere Textempfänger, die dieses okkasionelle Jugendwort nicht kennen, stellt die formale Auffälligkeit des Okkasionalismus eine Herausforderung dar, um diese Gelegenheitsbildung richtig zu interpretieren. Der Okkasionalismus يوم الأنتخة yawm 'alantahah wird mit dem ganzen ausführlichen Text verstanden; und zwar der Tag der Ruhe und Faulheit.

\subsection{Expressive Funktion}

Bei okkasionellen Bildungen handelt es sich grundsätzlich um Mittel zur Erzielung eines bestimmten kommunikativen Zieles (vgl. Fellner, 2003, S. 5). Sodann ist zu klären, dass die Okkasionalismen nicht lexikalisiert und nur für ein einziges Mal gebildet werden, da sie nur für den Zweck der Einmaligkeit benutzt werden und die Funktion haben, etwas Neues für ein einziges Mal zu benennen, um die Aufmerksamkeit der Menschen schneller zu erzeugen und ihr Staunen und ihre Neugierde zu verursachen. Dank ihrer appellativen und zweckorientierten Eigenschaft werden die Okkasionalismen in der Werbesprache gerne gebraucht, um bestimmte Wirkungen beim Rezipienten auszulösen (vgl. Elsen 2011, S. 90). Sie dienen einer stilistischen Funktion, indem eine bestimmte Einstellung des Sprechers ausgedrückt und ein bestimmter Effekt beim Rezipienten ausgelöst wird (vgl. Wanzeck, 2010, S. 39).

Im Gegensatz zu lexikalisierten, usuellen und damit als ,normgerecht' eingestuften Wörtern dienen okkasionelle Bildungen in vielfältiger Weise zur Aktivierung und Wahrnehmungssteuerung, indem sie gegen vorhandene Erwartungen und Schemavorstellungen vorstoßen und bei den Rezipienten gedankliche Wiedersprüche und Konflikte auslösen bzw. ihn überraschen. (Krieg, 2005, S. 91f)

Hiermit kann man ein gutes Beispiel anführen: Der Okkasionalismus das smett mir steht für ,das schmeckt mir ${ }^{6}$ und hat einen spielerischen Charakter, indem es sich auf (Hareico Smett) bezieht.

Frau: Ist der kleine schon in der Schule?

Mutter: Nein, er ist erst vier.

Der Kleine: mhhh das smett mir!

Frau: das heißt ,,schmeckt mir!“ “

Der Kleine: das smett mir!

Frau: schmeckt mir!

Der Kleine: smett! 
Frau: Nein!

Der kleine: kannst du nicht lesen ,,smett“ “

Frau: Oh!

Die Wurst im Frische-Brecher von Hareico

(vgl. das smett mir, 2010).

Das ist ein Werbespot von Hareico Smett am 06. Dezember 2010. Die Adressaten sind Allgemein-Fans/ Konsumenten. Der Werbespot zeigt einen Teil eines Gesprächs zwischen zwei Frauen zu einem Frühstück. Die Frau fragt ihre Freundin, ob ihr Sohn schon in die Schule geht, um lesen und schreiben zu lernen. Dann verneint die Mutter, da der Junge noch klein ist. Dazwischen gibt der Junge seiner Mutter einen Becher mit Streichwürsten, um ihm ein Sandwich zu machen. Wenn er es isst, sagt er „,das smett mir!“”. Die Frau denkt, dass der Junge nicht sprechen kann und versucht mit dem richtigen Satz ,das schmeckt mir!“ " zu korrigieren. Der Junge wirft bei dieser Gelegenheit eine okkasionelle Bildung ein, mit der er die Erwartungen der Frau verstößt, sie überrascht und bestenfalls auch provoziert. Diese starke Abweichung von Okkasionalismen durch Spiele auf Laut- und/oder Schreibebene macht von denen ein expressives stilistisches Sprachmittel. Darüber hinaus ist das Konversionsverb smett produktspezifisch, da es von einem Produktnamen abgeleitet wurde, und deswegen gebildet wurde, um eine spezifische Handlung aufzuzeigen; und zwar

die kognitive Verbindung zwischen Werbeslogan und Produkt herzustellen und damit positive Assoziationsketten zu schaffen. Das okkasionelle Verb suggeriert eine vollkommen neue Tätigkeit, was das Verlangen nach dem beworbenen Produkt steigern soll. Gleichzeitig wird durch die Konversion des Produktnamens zum „Tun-Wort” eine Legitimierung des Produkts vollzogen, da gezeigt wird, dass man damit eine spezielle Handlung vollziehen kann, die allen Verbrauchern, die das Produkt nicht erwerben, verwehrt bleibt. (Fellner, 2009, S. 51)

Dieser Okkasionalismus dient zur expressiv einschätzenden Bezeichnung bestimmten Produkts, beeinflusst die Meinung und bleibt dem Zuschauer aufgrund ihrer Einzigartigkeit im Kopf. Es löst beim Konsumenten bestimmte Einstellungen und auch bestimmte Handlungen aus, und zwar beispielsweise, um die Waren zu kaufen, was hauptsächlich im Interesse der Produzenten liegt.

Als Beispiel soll der Okkasionalismus ProBier's dienen. ProBier's 
besteht aus dem Präfix ,pro-” im Imperativverb probieren und dem Substantiv „Bier”. Es ist ein Werbeslogan für ein Biergetränk aus der Brauerei Stuttgarter Hofbräu und bezeichnet sich als eine Analogiebildung vom Imperativverb probier's! mit der Bedeutung: „durch eine Kostprobe den Geschmack von etwas prüfen, bevor man mehr davon isst oder trinkt" (DDU, 2007, S. 1320). Durch den Bedeutungswandel und die Auffälligkeit des Wortes fängt diese okkasionelle Bildung den Blick und erweckt die Aufmerksamkeit des Konsumenten, da die Erwartungshaltung des Konsumenten, der die lexikalisierte Bedeutung des Wortes erwartet, enttäuscht wird. Die Abweichung in der Orthografie ProBier's vom gewohnten Schriftbild probier's! dient der Funktion des „Blickfangs”, der die Aufmerksamkeit des Konsumenten auf die Wortbestandteile lenkt, um die alte Bedeutung des Wortes zu aktualisieren. Es beinhaltet eine positive Bewertung und eine indirekte Aufforderung, dieses Bier zu probieren.

Originelle und auffällige Neubildungen stellen damit eine wichtige Möglichkeit dar, mittels Sprache Aufmerksamkeit zu erregen und die Zielpersonen auf kognitivem Weg zu aktivieren. (Krieg, 2005, S. 92)

Wie gesehen, fordert dieser Vorgang mehr kognitive Leistungen von Zielpersonen; und zwar mit dem gezielten Text zu verbinden, ihre Aufmerksamkeit in Bezug auf den Textverlauf zu steuern, den Text gründlicher und vertiefter zu verarbeiten und ihr Interesse zu evozieren.

Viele neue Werbeslogns sind mitunter heutzutage in ägyptischen Werbungen mittels okkasionellen Bildungen konstruiert, wie اللحظة اللى مش على beispielsweise der Werbeslogan der bubbly-Schokolade allahẓa allī miš 'alā mazāgak bublilha (dt. Passt der Moment deiner Stimmung nicht, dann bubble ihn). Das ist ein Werbespot von Bubbly-Schokolade im Jahre 2018. Das neugebildete okkasionelle Wort verbalisiert, wie man gute Laune hat, wenn man Bubbly isst. An dieser Stelle muss man besonders betonen, dass das Verb ببلها bublilha die Gefühle der Rezipienten anspricht, indem das Wort Stimmung/Laune erwähnt wurde. Die Werbung vermarkt auf diese Weise kein Produkt, sondern vermarkt etwas für heitere Stimmung. Der Okkasionalismus ببلها bublilha unterstützt außerdem, dass der Rezipient den Produktnamen in seinem Gedächtnis behaltet. Wenn die okkasionellen Bildungen mit dem 
jeweiligen Produkt oder Produktnamen verschmolzen werden, schaffen sie Assoziationsnetzwerke, rufen das Interesse des Rezipienten hervor und steuern den Rezeptionsvorgang. Mit der Zeit üben sie die Erinnerungsfunktion, wenn der Produktname im Gedächtnis des Rezipienten bleibt.

Ein weiteres Beispiel für die ägyptischen Okkasionlismen in der Werbesprache ist der Werbeslogan اللى فات كاش واللى جاى ميزة allī fāt kāǎs w allì gāy mìza (dt. das Bargeld ist vorbei und Miza -Vorteil-Karte - ist kommend) كاش ولا ميزة kāš w lā mīza (dt. in bar oder mit Miza-Karte). Das Wort Miza wurde als Analogie zu dem bekannten Vorbild des Visa-Karte gebildet. Der Kontext spielt hier eine große Rolle beim Verstehen des Okkasionalismus, wobei das Wort كاش $k \bar{a} s ̌$ (dt. in bar) als Gegenteil des Kreditkarten eingeführt wurde. In Unabhängigkeit von diesem gewissen Kontext wird das lexikalisierte Wort ميزة miza als Vorteil verstanden, und nicht als ein positives leichtes bargeldloses Zahlungsmittel. Dieser spielerische Charakter des Okkasionalismus bewirkt auf diese Weise die Spannung beim Rezipienten und übt die Gelegenheitsbildung eine Attraktivitätsfunktion aus.

\section{Fazit}

Aus den bisherigen Ausführungen lässt sich die Schlussfolgerung ziehen, dass die Okkasionalismen nur für eine bestimmte kommunikative Gelegenheit geschaffen werden und ihren Sinn nur im jeweiligen Kontext erhalten. Die Termini Okkasionalismus, Neologismus und usuelle Wörter wurden ebenfalls in diesem Beitrag erörtert und voneinander abgegrenzt. Die Frage dieses Beitrags war die Suche nach den Ursachen des Gebrauchs der Okkasionalismen. Ursachen bzw. Funktionen der Okkasionalismen bestehen darin, eine sprachliche Lücke zu füllen, das Interesse an etwas zu erhöhen und bestimmte Informationen über die Produkte hervorzuheben sowie stilistische und spielerische Intentionen zu verwirklichen. Man kann also zum Schluss kommen, dass diese neuen Wortbildungen ein ausgezeichnetes Mittel sind, sich an kulturellen, sozialen, ökonomischen, politischen und technischen Veränderungen und an dem Fortschritt in den einzelnen Fachwissenschaften anzupassen. In diesem Beitrag wurde auf einige Aspekte der Auseinandersetzung mit 
dem Phänomen Okkasionalismen im deutschen und ägyptischarabischen Sprachgebrauch eingegangen. Es wurde ebenfalls ein Versuch unternommen, den Gebrauch der okkasionellen Bildungen darzustellen und auf die wichtigsten Aspekte ihrer Auswirkungen und Funktionen hinzuweisen. Die Funktion der Okkasionalismen hängt von der Situation bzw. dem Kontext ab, in denen sie erscheinen. Die Okkasionalismen dienen nicht nur der Benennungsfunktion, indem sie Gegenstände und Sachverhalte benennen oder spezifizieren, sondern sie werden ebenfalls in der Werbesprache in beiden Sprachen immer wieder gebraucht werden, um bestimmte Wirkungen und Effekte beim Konsumenten zu veranlassen. Ihre Hauptfunktion in diesem Bereich ist es, ein Produkt so attraktiv wie möglich zu präsentieren, um den Verkauf des Produktes zu erhöhen. Daraus kann man schließen, dass die deutschen okkasionellen Bildungen in Werbungen den Rezipienten zum Nachdenken (z.B. smett mir) veranlassen, wohingegen sind die ägyptisch-arabischen Okkasionalismen in den Werbetexten oft emotional gestaltet (z.B. ببلها bublilha). Die textspezifische Rolle der Okkasionalismen besteht darin, andere komplexe und lange Wörter zu ersetzen. Der Gebrauch der Okkasionalismen fordert mehr kognitive Leistungen von Rezipienten; und zwar mit dem gezielten Text abzuhängen, um diese Gelegenheitsbildungen richtig zu verstehen. Zusammenfassend lässt sich feststellen, dass das Deutsche und das Ägyptisch-Arabische solche okkasionelle Wörter brauchen und verwenden, weil die Okkasionalismen insbesondere die Entwicklung und die Modernisierung der Welt und des Lebens reflektieren.

\section{Anmerkungen}

i. Dieses Beispiel stammt aus der Webseite

https://www.inhaltsangabe.de/wissen/stilmittel/neologismus /, abgerufen am 27/07/2020.

ii. vgl. dazu das Wort des Jahres der Gesellschaft für deutsche Sprache: https://gfds.de/aktionen/wort-des-jahres/ \#, abgerufen am 31/07/2020.

iii. vgl. ebd.

iv. Für weitere Beispiele s. die Webseite: https://bit.ly/3o16VII, abgerufen am 1/9/2020.

v. https://bit.ly/2JgkjtJ, abgerufen am 30/8/2020.

vi. https://bit.ly/3mm5WTa, abgerufen am 30/8/2020.

vii. $\quad$ vgl. dazu https://bit.ly/37ep9Q9, abgerufen am 30/8/2020. 


\section{Zitierte Literatur}

Barz, I. (2007). Wortbildung - praktisch und integrativ: Ein Arbeitsbuch (Vol. 2). Frankfurt am Main: Peter Lang.

Bußmann, H. (Hg.) (2008). Lexikon der Sprachwissenschaft. Stuttgart: Kröner.

Das smett mir (2010). Abgerufen vom: https://bit.ly/39oK5VE. Abgerufen am 29. Juli 2020.

Duden Universalwörterbuch. (2007). Mannheim: Bibliographisches Institut GmbH.

Dudenredaktion (Hsg.) (2009). Die Grammatik. 8., überarbeitete Auflage. Mannheim: Bibliographisches Institut GmbH.

Elsen, H. (2011). Neologismen: Formen und Funktionen neuer Wörter in verschiedenen Varietäten des Deutschen. 2., überarbeitete Auflage. Tübingen: Narr.

Fellner, R. (2009). Okkasionalismen in Werbeslogans zwischen 2003 und 2008 unter besonderer Berücksichtigung der Branchen Kosmetik, Ernährung, Getränke und Pharmazie. München:Grin Verlag GmbH.

Glück, H. \& Rödel, M. (Hg.). (2005). Metzler Lexikon Sprache. 3., Neubearb. Aufl. Unter Mitarbeit v. Friederike Schmöe. Stuttgart: Metzler.

Harm, V. (2015). Einführung in die Lexikologie. Darmstadt: WBG.

Krieg, U. (2005). Wortbildungsstrategien in der Werbung: Zur Funktion und Struktur von Wortneubildungen in Printanzeigen. Hamburg: Buske.

Matussek, M. (1994). Wortneubildungen im Text. Hamburg: Buske.

Ortner, H. \& Ortner, L. (1984). Zur Theorie und Praxis der Kompositaforschung. Mit einer ausführlichen Bibliographie. Tübingen: Narr.

Ulrich, W. (2002). Wörterbuch linguistische Grundbegriffe. 5. Auflage. Berlin \& Stuttgart: Gebrüder Borntraeger.

Wanzeck, C. (2010). Lexikologie: Beschreibung von Wort und Wortschatz im Deutschen. Göttingen: Vandenhoeck \& Ruprecht.

Zeitmagazin (2017). Hammerkörper in acht Wochen. Abgerufen vom: http://bit.ly/39lqtBP. 14. Juli 2017. 